\title{
Mismatch Repair
}

National Cancer Institute

\section{Source}

National Cancer Institute. Mismatch Repair. NCI Thesaurus. Code C20639.

The mismatch repair (MMR) system promotes genomic fidelity by repairing base-base mismatches, insertion-deletion loops and heterologies generated during DNA replication and recombination. This function is critically dependent on the assembly of multimeric complexes involved in mismatch recognition and signal transduction to downstream repair events. 